\title{
Simulation of the movement behavior of Leopardus guigna using random walkers
}

\author{
A. Torres-Hernandez ${ }^{*}, \mathrm{a}$, Byron C. Guzmán Marín ${ }^{\dagger, b}$, Melanie Kaiser ${ }^{\ddagger}, \mathrm{b}$, and Julio C. Hernández-Hernández $\$, c$ \\ ${ }^{a}$ Department of Physics, Faculty of Science - UNAM, Mexico \\ ${ }^{b}$ Foundation for Wild Cat Coordination, Santiago, Chile \\ ${ }^{c}$ Pronatura Veracruz A. C., Mexico
}

\begin{abstract}
Scarcity of information regarding the natural history of Leopardus guigna, an endangered felid native to Chile and western Argentina, hampers the successful implementation of conservation strategies. Very little is known about the movement behavior of L. guigna and home range estimates rest on less than a handful of radiotracking studies. Due to the difficulties inherent in any study requiring the live capture of protected species, we suggest a mathematical approach to the modeling of territories occupied by individual guignas. Our approach is based on the hypothesis that L. guigna maintains a sedentary behavior in a specific area of a given territory, whereby starting points can be derived from point observations. In this context, the present article shows a way to simulate a distribution of points in a territory using random walkers to emulate field data as could be obtained by means of telemetry. Our model allows for an estimation of mean distances covered by individual cats from a fixed starting point and thus enables the prediction of interactions with known threats to the species, such as motor vehicles, free-roaming dogs, and people. The knowledge gained in this process can be of much help when defining the boundaries of protected areas and, more generally, in the planning of infrastructure, rural and urban development.

Keywords: movement behavior, behavioral modeling, random walkers, iteration function, pseudorandom numbers.
\end{abstract}

\section{INTRODUCTION}

Leopardus guigna is the smallest wild cat in the Neotropics and has a very narrow distribution range. It is endemic to central and southern Chile and a thin strip of Western Argentina. In detail, northernmost observations of this species have been made in the Chilean Coquimbo Region, at about $31^{\circ}$ South, while the southern limit of its known distribution range is in the Aysén Region of Chile, at $48^{\circ}$ South [1]. This area has long since been characterized by vast native forests that provided ample space to these territorial cats. Unfortunately though, extensive changes of land use have led to the degradation and loss of large parts of the guigna's habitat. Deforestation and parcelling of hitherto intact areas is putting pressure on guigna populations, especially in the southern part of their range [2]. What's more, these actions precede the presence of people, domestic animals, and motor vehicles, which constitute the main threats to L. guigna and account for significant anthropogenic mortality. As of today, the species is classified as Vulnerable both by the International Union for Conservation of Nature and Chilean species classification regulations [3]. A national guigna conservation plan was launched in 2017; it recognizes the urgent need to generate scientific and technical information in order to strengthen the authorities' capacity to manage the species and its ecosystem.

Because the reliable prediction of individual guignas' movements may support the successful design of mitigation strategies, data on sighting records, home ranges, and movement behavior of this species should be taken into account during the decision-making on urban planning, road building, and the designation of protected areas. There are several reasons why this is not currently done satisfactorily, not the least of which is the lack of population and behavioral data.

\footnotetext{
${ }^{*}$ Email: anthony.torres@ciencias.unam.mx; ORCID: 0000-0001-6496-9505

${ }^{\dagger}$ Email: b.guzman.marin@outlook.com; ORCID: 0000-0001-7051-1154

‡Email: melanie.kaiser.pv@gmail.com; ORCID: 0000-0002-8592-926X

§Email: biol.julio@gmail.com; ORCID: 0000-0002-1286-2404
} 
One of the few studies on L. guigna home ranges was carried out in the fragmented landscape of the Chilean Araucanía Region and found that these cats had mean 95\% usage areas of $6.23 \pm 4.00 \mathrm{~km}^{2}[4]$. The authors related their findings to a prior publication, where smaller home ranges were reported for guignas living in two large, pristine national parks (mean 90\% kernel home range $=1.19 \mathrm{~km}^{2} ;[5]$ ). They hypothesized that guignas may increase their home range to compensate for lesser forest coverage [4]. Notwithstanding, Sanderson and colleagues reported home ranges of $0.8-3.7 \mathrm{~km}^{2}$ for guignas living in highly fragmented areas and observed two males occupying $1.8 \mathrm{~km}^{2}$ and $22.4 \mathrm{~km}^{2}$, respectively, in a contiguous forest with less human influence [6].

These data on L. guigna home ranges have been acquired by means of radiotracking studies, which imply the live capture and anesthesia of individual cats, their fitting with radiocollars and subsequent release. Beyond the direct impact on target populations, this method is labor intensive and usually requires the presence of people in the field to monitor the signals emitted by the trackers. Notwithstanding, valuable data on activity patterns, movement behavior, and even intraspecies interactions may be gathered. In the present article, we propose a mathematical approach to obtain such data, thereby complementing field work and reducing the need of wildlife manipulation. Our approach is based on the main findings of prior studies on guignas' movement behavior, as summarized by Sanderson et al. [6]:

" ... habitat fragmentation and loss may affect male and female guignas differently. Females were sedentary and rarely explored areas beyond their established home ranges. In contrast, males constantly moved about their territories, presumably marking their boundaries and visiting females. ... Males were therefore more likely to come into contact with humans, their pets, and domestic fowl."

The fact that females rarely explore beyond a rather small home range within their habitat could be interpreted as an evolutionary response to the presence of predators and other types of threats. In modern times, it may also help to avoid hazardous contact to men and domestic animals. It thus seems feasible that male guignas may adopt a similarly sedentary behavior to increase their chances of survival in a highly intervened environment. We thus developed our model under the hypothesis that guignas maintain a sedentary behavior in a specific area of a given territory and show a way to simulate the guignas' movement as a distribution of points in that territory. In detail, we use random walkers to emulate radiotracking data to estimate mean distances covered by individual guignas while moving from a certain fixed position. These estimates may be used to predict the likelihood of encounters between L. guigna, motor vehicles, humans, free-ranging domestic dogs and cats as well as poultry.

\section{RANDOM WaLKers}

Denoting by $\left\{\hat{e}_{k}\right\}_{k=1}^{n}$ the canonical basis of $\mathbb{R}^{n}$. So, if $x \in \mathbb{C}^{n}$, it may be written using Einstein notation as follows

$$
x=\hat{e}_{k}[x]_{k}=\hat{e}_{k} \operatorname{Re}\left([x]_{k}\right)+i \hat{e}_{k} \operatorname{Im}\left([x]_{k}\right),
$$

where $[x]_{k}$ denotes the $k$-th component of vector $x$. On the other hand, considering a set $\Omega_{z} \subset \mathbb{Z}$, defined as follows

$$
\Omega_{z}:=\{-z,-(z-1), \cdots, z-1, z\},
$$

it is possible to define the following set of vectors

$$
\mathcal{S}\left(\mathbb{C}^{n}, \Omega_{z}\right):=\left\{s \in \mathbb{C}^{n}: \operatorname{Re}\left([s]_{k}\right), \operatorname{Im}\left([s]_{k}\right) \in \Omega_{z} \forall k \geq 1\right\} .
$$

So, denoting by card $(\cdot)$ the cardinality of a set, it is possible to obtain the following results:

$$
\text { If } \operatorname{card}\left(\Omega_{z}\right)=k \Rightarrow \operatorname{card}\left(\mathcal{S}\left(\mathbb{C}^{n}, \Omega_{z}\right)\right)=2 k^{n} \text { and } \operatorname{card}\left(\mathcal{S}\left(\mathbb{R}^{n}, \Omega_{z}\right)\right)=k^{n} \text {, }
$$

$$
\text { If } \operatorname{card}\left(\Omega_{z}\right)=k \Rightarrow \exists M>0 \text { such that }\|s\| \leq M \forall s \in \mathcal{S}\left(\mathbb{C}^{n}, \Omega_{z}\right) \text {. }
$$



follows

Therefore, considering a value $r>0$ and some set $\mathcal{S}\left(\mathbb{C}^{n}, \Omega_{z}\right)$, it is possible to define a function $\Phi_{r}: \mathbb{C}^{n} \rightarrow \mathbb{C}^{n}$ as

$$
\Phi_{r}(x):=x+r s
$$

with which it is possible to define the following iterative method

$$
x_{i+1}:=\Phi_{r}\left(x_{i}\right)=x_{i}+r s_{i}, \quad i=0,1,2, \cdots .
$$

So, considering the following set

$$
B\left(x_{0} ; R\right):=\left\{x:\left\|x_{0}-x\right\|<R\right\},
$$

and allowing the vectors $s_{i}$ to be chosen randomly from the set $\mathcal{S}\left(\mathbb{C}^{n}, \Omega_{z}\right)$, it is possible to obtain the following result (see Figure 1):

$$
\forall x_{0} \in \mathbb{C}^{n} \exists R=R(r)>0 \text { such that }\left\{x_{i}\right\}_{i=1}^{M} \in B\left(x_{0} ; R\right),
$$

where $\left\{x_{i}\right\}_{i=1}^{M}$ denotes a finite sequence of random variables generated through the function $\Phi_{r}$, which may be named an iteration function of a random walker. It is necessary to mention that the sequence $\left\{x_{i}\right\}_{i=1}^{M}$ defines a trajectory that may be termed as the trajectory of a random walker, while the value $r$ may be termed as the step size of a random walker.

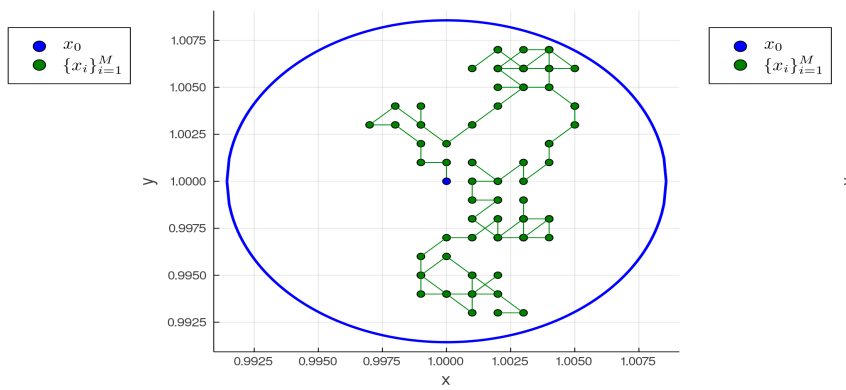

a) $r=0.001$

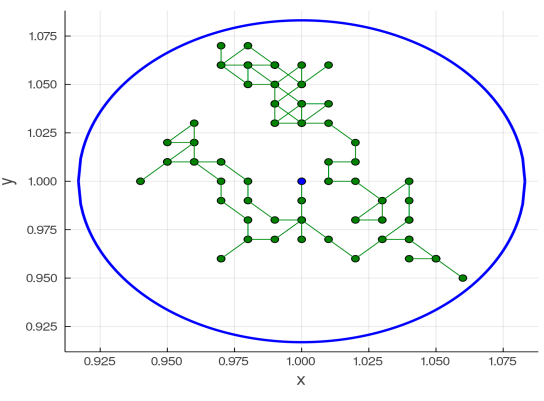

b) $r=0.01$

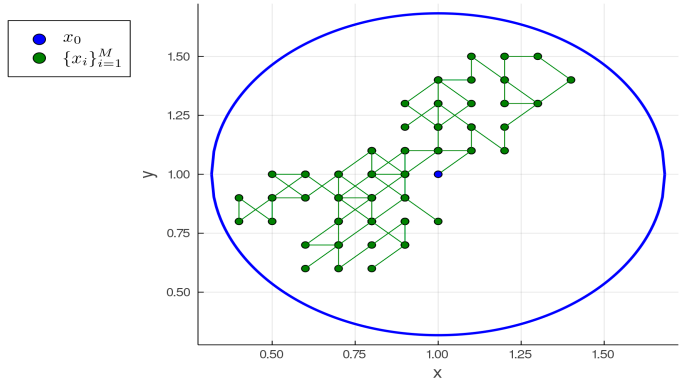

c) $r=0.1$

Figure 1: Illustrations of some trajectories of random walkers in $\mathbb{R}^{2}$ for the same initial condition $x_{0}$ but with different step sizes $r$ using the set $\mathcal{S}\left(\mathbb{R}^{2}, \Omega_{1}\right)$.

Defining the following set

$$
\operatorname{Gen}_{M}\left(x_{0}\right):=\left\{\Phi_{r}:\left\{\Phi_{r}\left(x_{i}\right)\right\}_{i=0}^{M-1} \in B\left(x_{0}, R\right)\right\},
$$

which may be interpreted as the set of iteration functions of random walkers defined in some set $\mathcal{S}\left(\mathbb{C}^{n}, \Omega_{z}\right)$, which using the initial value $x_{0}$ generate a finite sequence of random variables $\left\{x_{i}\right\}_{i=1}^{M} \in B\left(x_{0} ; R\right)$. So, considering the result of the equation (7), it is possible to obtain the following corollary (see Figure 2): 
Corollary 1. Let $\Phi_{r}: \mathbb{C}^{n} \rightarrow \mathbb{C}^{n}$ be an iteration function of a random walker defined in some set $\mathcal{S}\left(\mathbb{C}^{n}, \Omega_{z}\right)$. So, for any sequence $\left\{x_{i}\right\}_{i \geq 1} \in \mathbb{C}^{n}$, it is fulfilled that

$$
\Phi_{r} \in \operatorname{Gen}_{M}\left(x_{j}\right) \forall x_{j} \in\left\{x_{i}\right\}_{i \geq 1},
$$

and therefore

$$
\forall x_{j} \in\left\{x_{i}\right\}_{i \geq 1} \exists B\left(x_{j} ; R_{j}\right) .
$$

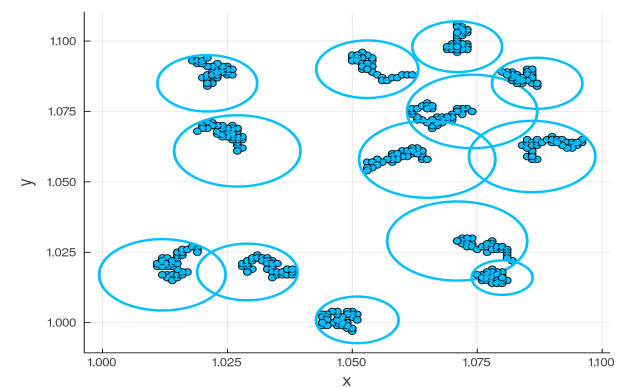

a) $r=0.001$

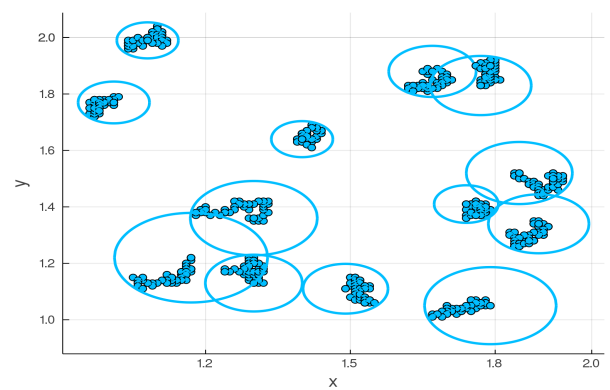

b) $r=0.01$

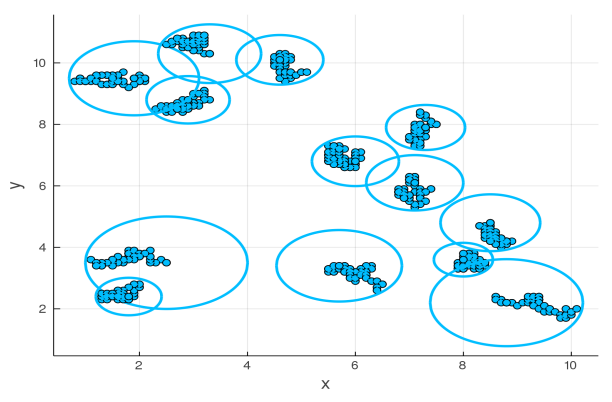

c) $r=0.1$

Figure 2: Illustrations of some trajectories of random walkers in $\mathbb{R}^{2}$ for different initial conditions $\left\{x_{i}\right\}_{i=1}^{13}$ and different step sizes $r$ using the set $\mathcal{S}\left(\mathbb{R}^{2}, \Omega_{1}\right)$.

\section{Proposed Model}

From the previous section, it is possible to obtain the following observations:

i) Any random walker may be considered as an iterative method through an iteration function $\Phi_{r}$, defined on a set $\mathcal{S}\left(\cdot, \Omega_{z}\right)$ with a fixed step size $r$.

ii) Any random walker may be characterized by its initial position $x_{0}$ and its iteration function $\Phi_{r}$, which by generating a finite trajectory, generates a finite sequence of random variables $\left\{x_{i}\right\}_{i=1}^{M}$.

iii) For any random walker that generates a finite sequence of random variables $\left\{x_{i}\right\}_{i=1}^{M} \in B\left(x_{0} ; R\right)$, there exists an associated radius $R_{M}<R$, such that

$$
\left\|x_{0}-x_{j}\right\| \leq R_{M} \forall x_{j} \in\left\{x_{i}\right\}_{i=1}^{M},
$$

where for an arbitrary positive constant $k$, it is fulfilled that

$$
R_{M}=k r \text {. }
$$

iv) Any random walker that generates a finite sequence of random variables $\left\{x_{i}\right\}_{i=1}^{M}$, may be characterized by its initial position $x_{0}$ and its associated radius $R_{M}$.

$v$ ) The behavior of a random walker, without considering external forces, is invariant of the scale. 
Applied Mathematics and Sciences: An International Journal (MathSJ) Vol.8, No.2/3/4, December 2021

So, considering the previous observations, it is possible to abstract the behavior of a sedentary animal as an iterative method that starts from a fixed initial condition (such as a den) and that generates a random trajectory (e.g., when searching for food in a specific area of a given territory) which is bounded by a circumference (see Figure 3). The previous description coincides with a random walker, but to generate data through random walkers that may be useful to simulate the behavior of L. guigna, to estimate mean distances traveled in a suitable unit of measure, and to predict the probability of encounters with direct threats, it is necessary to have real initial positions in a region of interest where the presence of L. guigna is confirmed. The latter may be achieved by means of camera traps or direct observations. On the other hand, and for the simulation presented herein, initial positions may be generated considering regions with possible guigna presence or through pseudorandom numbers that may serve as test data.
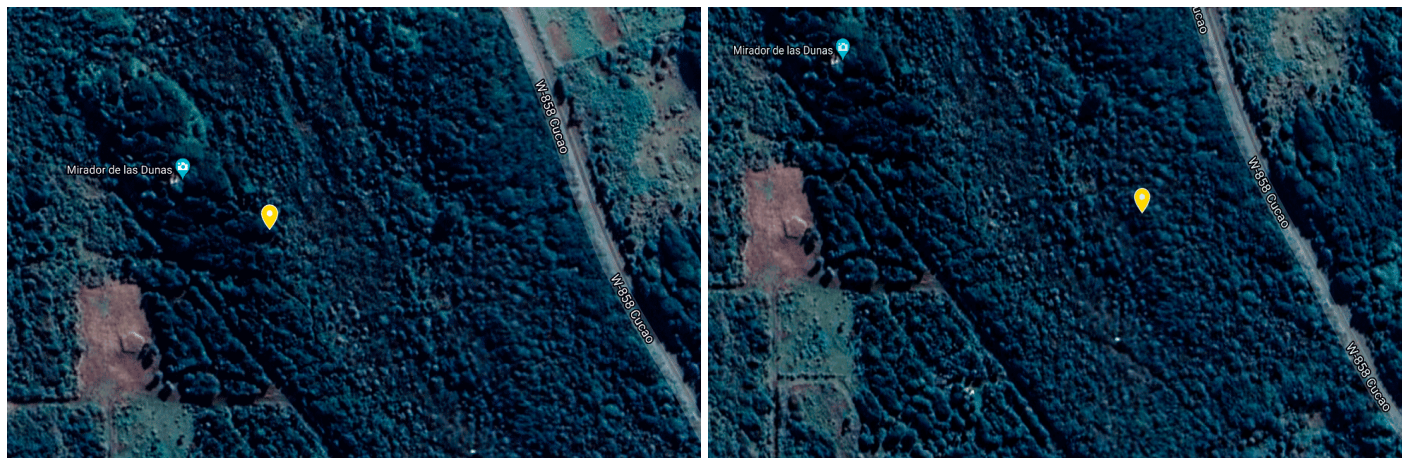

a) Identification of possible regions of interest
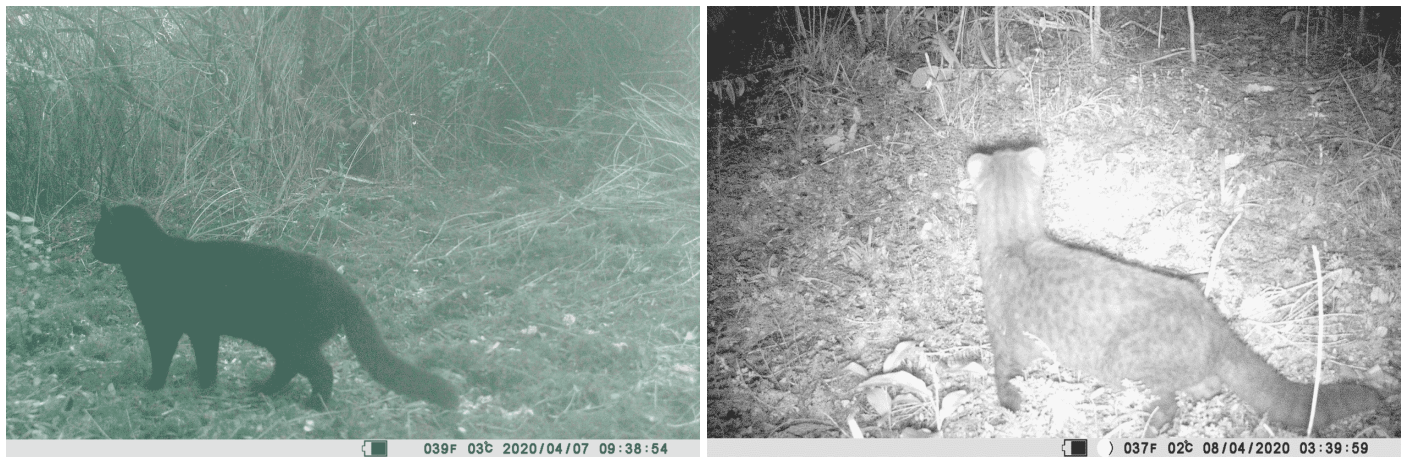

b) Confirmation of L. guigna presence in trap camera images
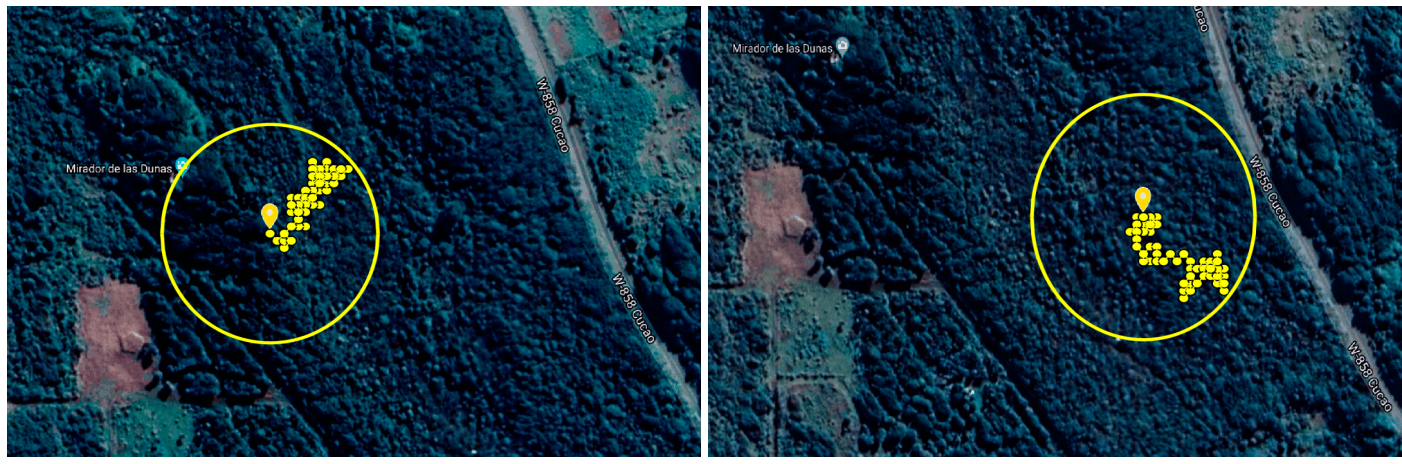

c) Simulation of L. guigna movement using random walkers

Figure 3: Steps towards the simulation of L. guigna movement behavior in specific areas using random walkers. Results as displayed in $c$ ) are comparable to data as could be obtained by telemetry studies in the field (see reference [7]). 


\subsection{EXAMPLE}

Before continuing it is necessary to define the following set:

Predators $:=\{x: x$ represents any external agent that may cause mortality of L.guigna $\}$,

with which it is possible to generalize all the points of a territory that are potentially fatal for L. guigna, such as urban areas, roads, and free-roaming domestic dogs, among others.

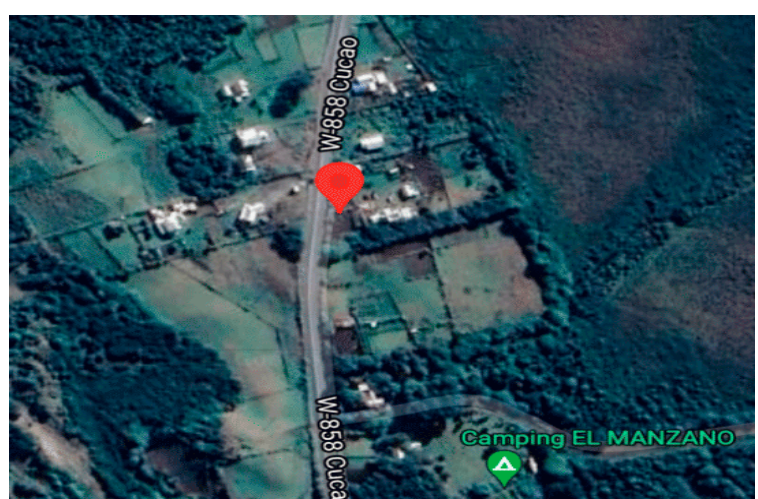

a) Urban areas
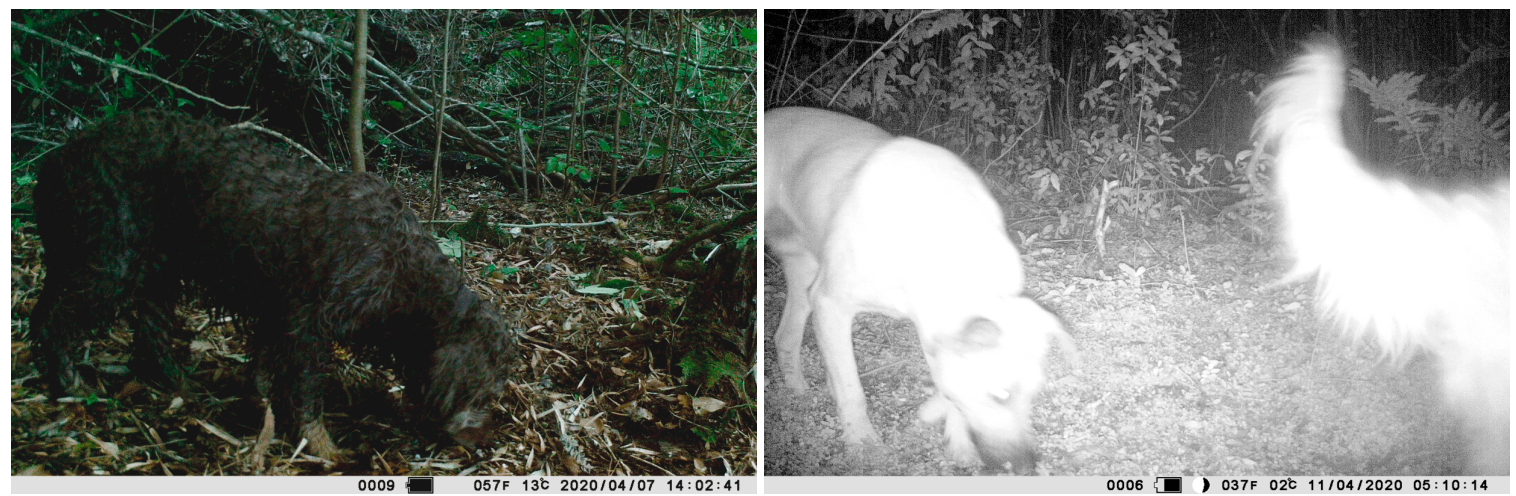

c) Free-roaming domestic dogs

Figure 4: Images of some elements belonging to the set Predators.

To carry out the simulation, it is necessary to generate a record of real positions of points belonging to the set Predators in a given territory with the presence of L. guigna (see Table 1 ).

\begin{tabular}{c|cc}
\hline Predators & Latitude & Longitude \\
\hline Predator 1 & -42.6149730 & -74.1147730 \\
Predator 2 & -42.6208730 & -74.1121220 \\
Predator 3 & -42.6228700 & -74.1112320 \\
Predator 4 & -42.6213250 & -74.1049990 \\
Predator 5 & -42.6142930 & -74.1153090 \\
Predator 6 & -42.6219000 & -74.1115460 \\
Predator 7 & -42.6160470 & -74.1140780 \\
Predator 8 & -42.6194878 & -74.1129435 \\
Predator 9 & -42.6181298 & -74.1137374 \\
Predator 10 & -42.6171034 & -74.1145528 \\
Predator 11 & -42.6161402 & -74.1119564 \\
Predator 12 & -42.6164560 & -74.1087378 \\
Predator 13 & -42.6147822 & -74.1092313 \\
Predator 14 & -42.6161244 & -74.1059483 \\
\hline
\end{tabular}

Table 1: Coordinates of the predators. 

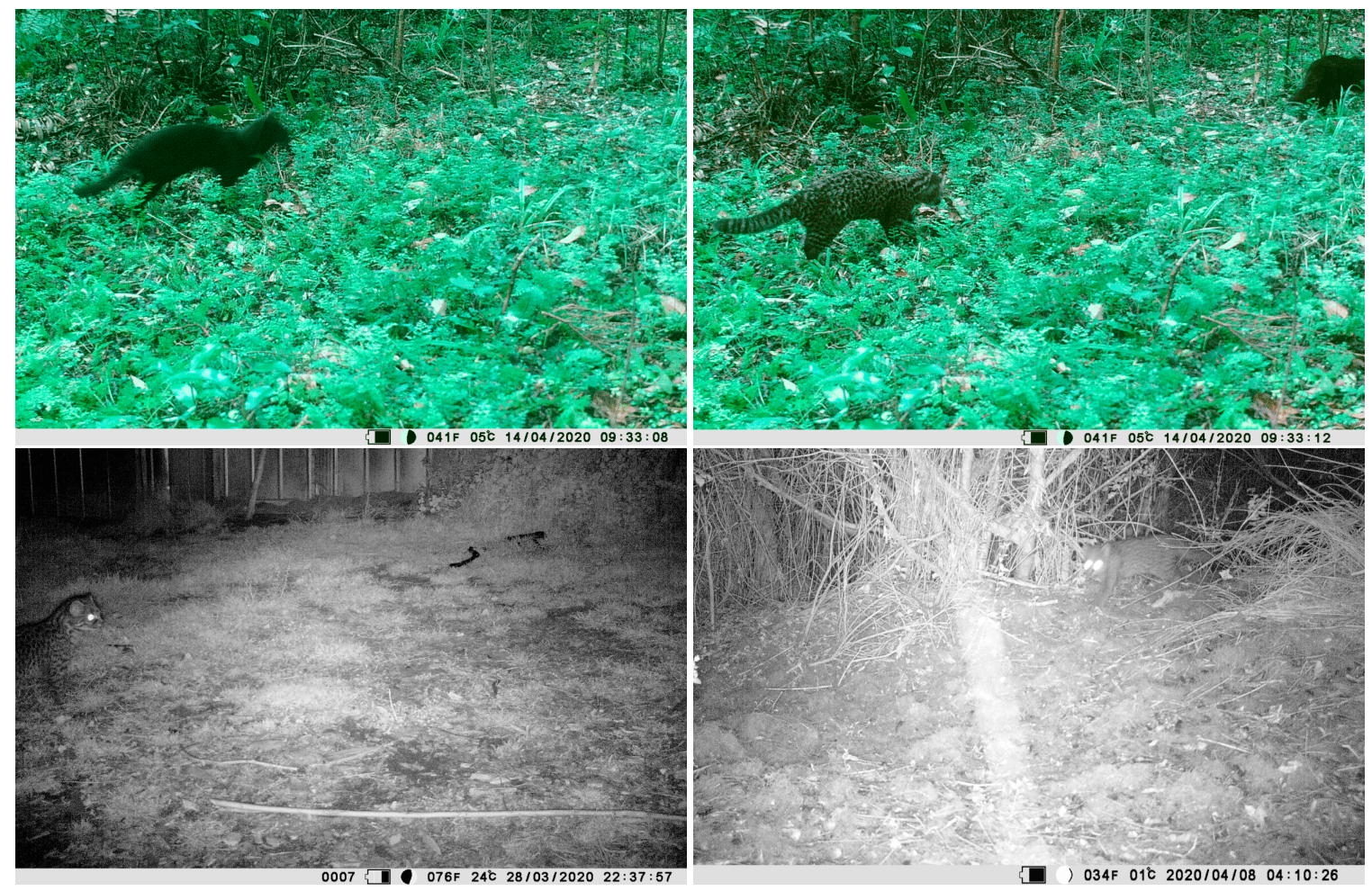

Figure 5: L. guigna in the territory of interest for the simulation.

Analogously, it is necessary to generate a record of real positions of points representing potential territories of L. guigna, which may be obtained through camera trap records (see Figure 5). However, in the absence of records confirming the presence of L. guigna, test data generated through pseudorandom numbers may be used. Due to the small number of available records, a combination of real data and test data is used in Table 2 .

\begin{tabular}{c|cc}
\hline Guignas & Latitude & Longitude \\
\hline Guigna 1 & -42.6215620 & -74.1139580 \\
Guigna 2 & -42.6220470 & -74.1124180 \\
Guigna 3 & -42.6185106 & -74.1069997 \\
Guigna 4 & -42.6174691 & -74.1117204 \\
Guigna 5 & -42.6211949 & -74.1090382 \\
Guigna 6 & -42.6166947 & -74.1166342 \\
Guigna 7 & -42.6145629 & -74.1123856 \\
Guigna 8 & -42.6202633 & -74.1143812 \\
Guigna 9 & -42.6190475 & -74.1099823 \\
\hline
\end{tabular}

Table 2: Coordinates of the guignas.

Using the coordinates of the predators and of the guignas, the distribution of points that will be analyzed in the simulation is generated with the help of a satellite image (see Figure 6). So, a numerical scale is assigned in the satellite image and the values that will be used to generate the iteration function of the random walkers are specified, it is necessary to mention that for this example the following values were chosen:

$$
r=5, \quad z=2, \quad M=200,
$$

with which the following iteration function is obtained to generate the simulation of the behavior of a population of guignas in a given region through random walkers:

$$
\Phi_{5}(x)=x+5 s \text { with } s \in \mathcal{S}\left(\mathbb{R}^{2}, \Omega_{2}\right) \text { and } \Phi_{5} \in \operatorname{Gen}_{200}\left(x_{0}\right),
$$

for each initial position $x_{0}$ that represents an individual guigna. 
Applied Mathematics and Sciences: An International Journal (MathSJ) Vol.8, No.2/3/4, December 2021

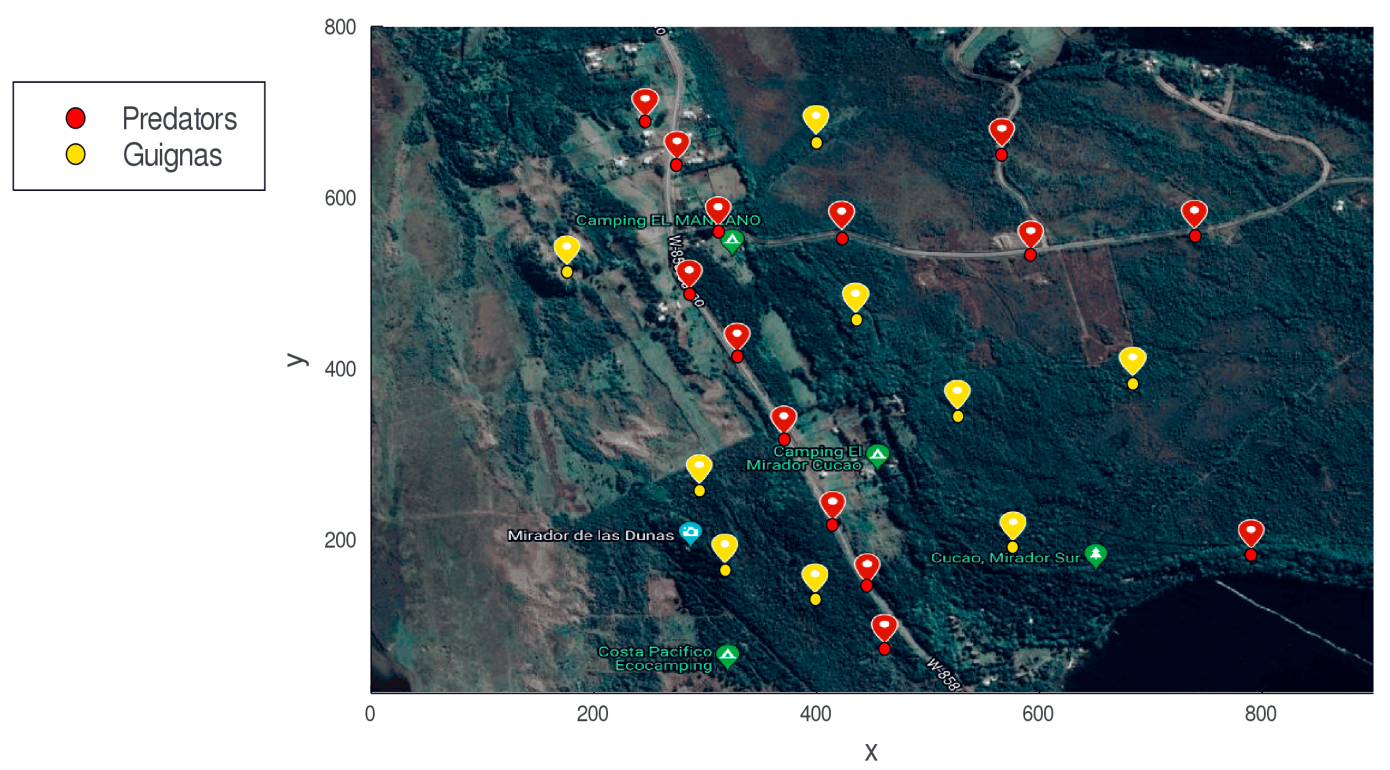

a) Generating a distribution of predators and guignas in a region of interest

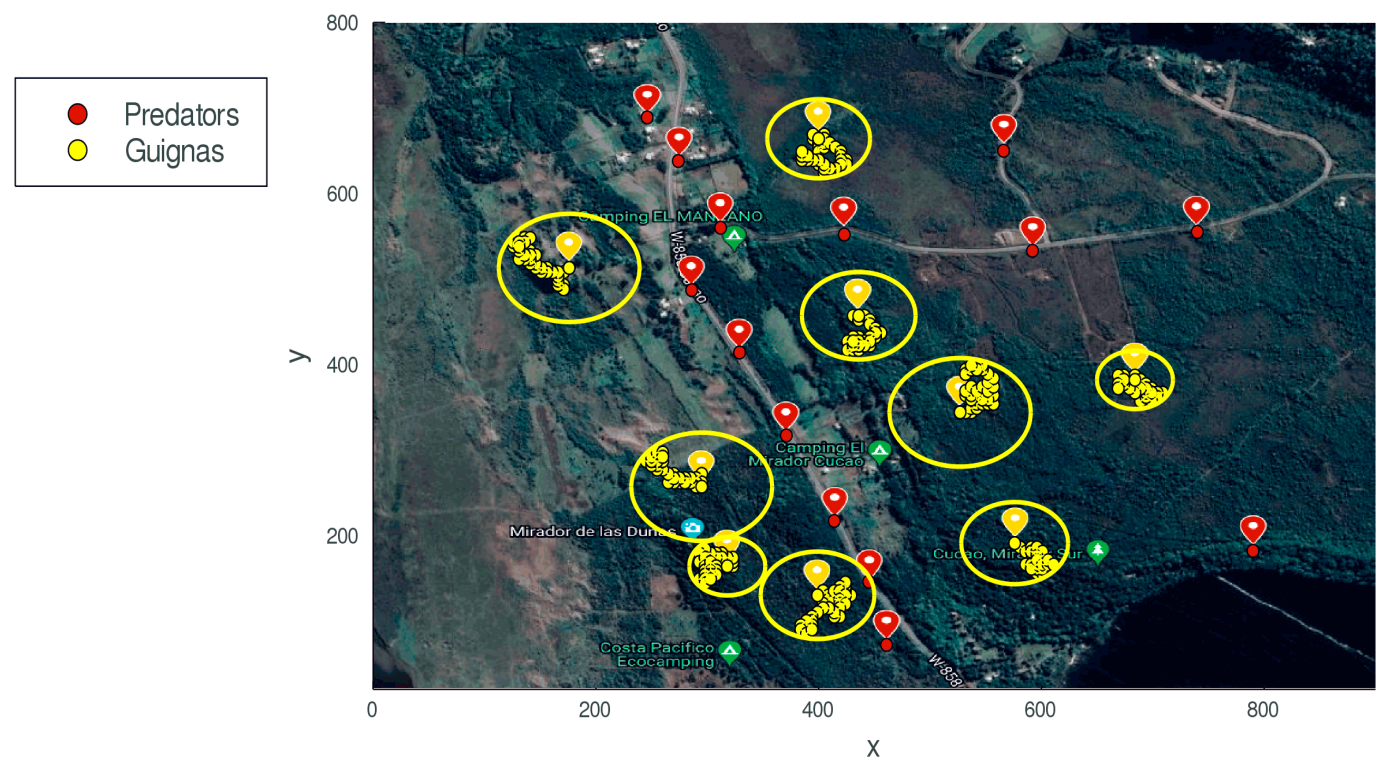

b) Simulation of L. guigna movement behavior using random walkers

Figure 6: Summary of the steps to follow to generate a simulation of a population of guignas in a given region through random walkers.

So, since $\Phi_{r} \in \operatorname{Gen}_{200}\left(x_{0}\right)$, for each initial condition $x_{0}$ it is possible to generate a finite trajectory of random variables $\left\{x_{i}\right\}_{i=1}^{200}$ that emulates the behavior of an individual guigna when looking for food in its territory. On the other hand, considering that the behavior of random walkers is invariant of the scale when external forces are not considered, it is more convenient to work with a scale of distances that depends on the step size $r$ chosen for the random walkers (see Figure 7, which may be obtained through the following result:

$$
\text { If } \Phi_{r} \in \operatorname{Gen}_{M}\left(x_{0}\right) \Rightarrow \forall x_{j} \in\left\{x_{i}\right\}_{i=1}^{M} \exists k_{j}=\frac{\left\|x_{0}-x_{j}\right\|}{r},
$$

where $k_{j}$ represents the number of steps $r$ that a random walker must travel in a straight line to get from the initial position $x_{0}$ to the position $x_{j}$. 

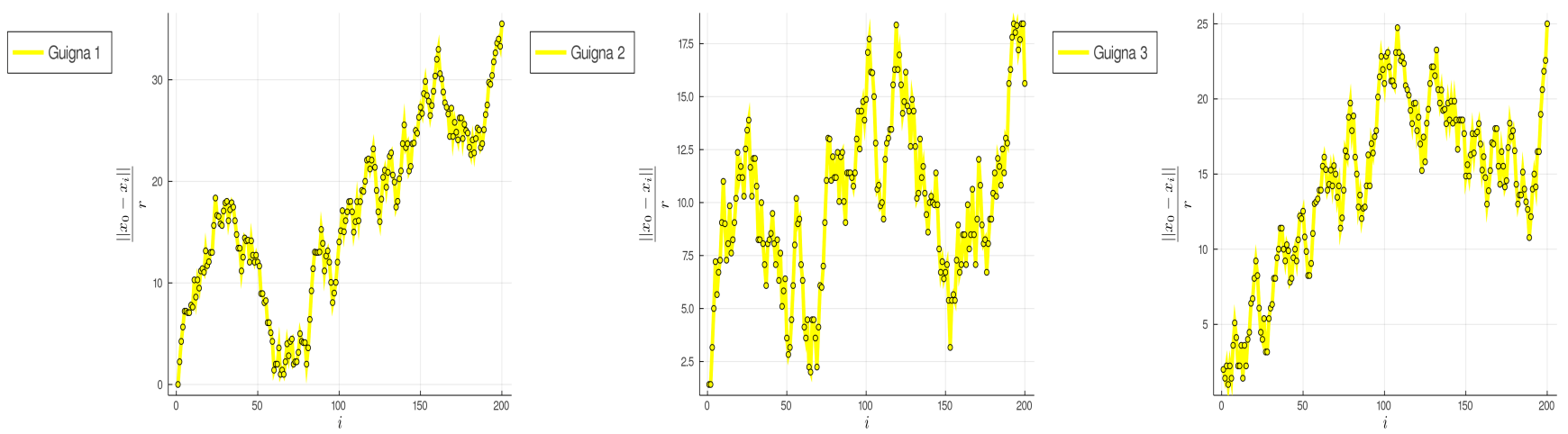

a) Sequence of distances for guigna 1

b) Sequence of distances for guigna 2

c) Sequence of distances for guigna 3
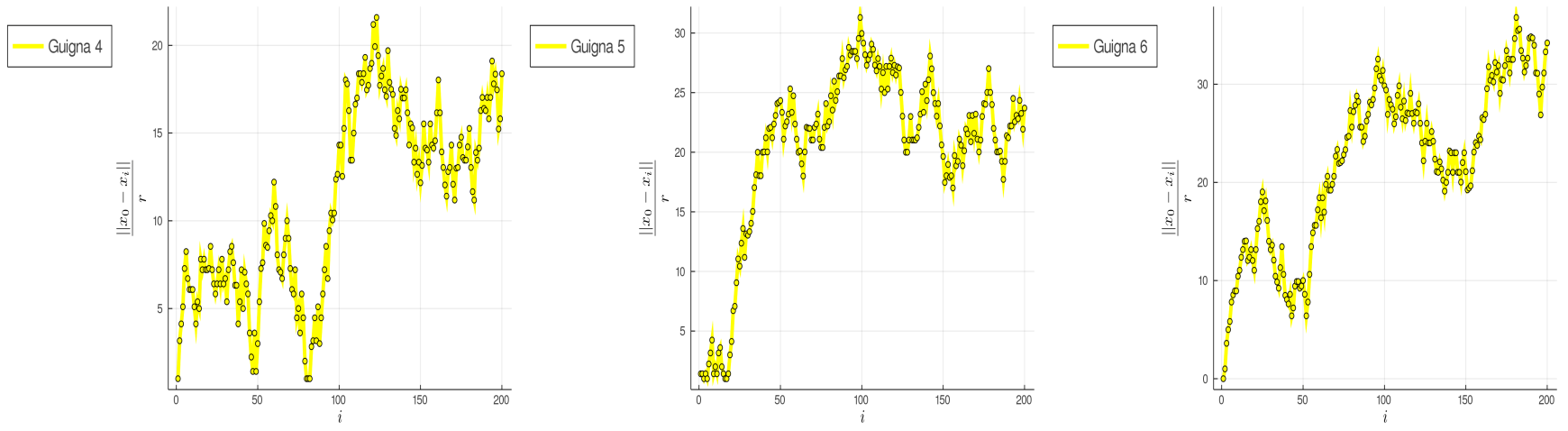

d) Sequence of distances for guigna 4

e) Sequence of distances for guigna 5

f) Sequence of distances for guigna 6

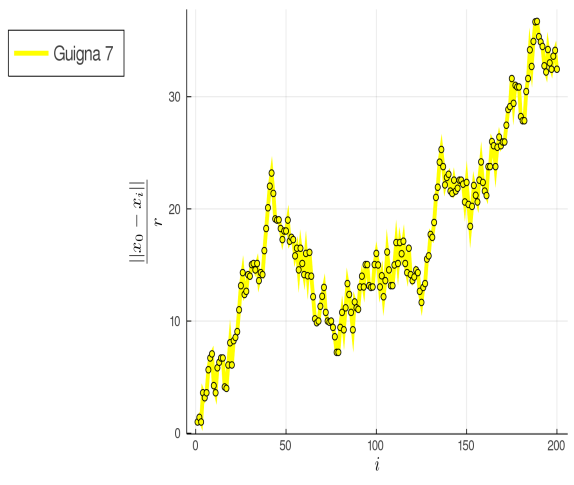

g) Sequence of distances for guigna 7

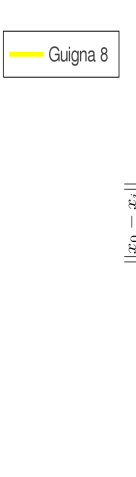

h) Sequence of distances for guigna 8

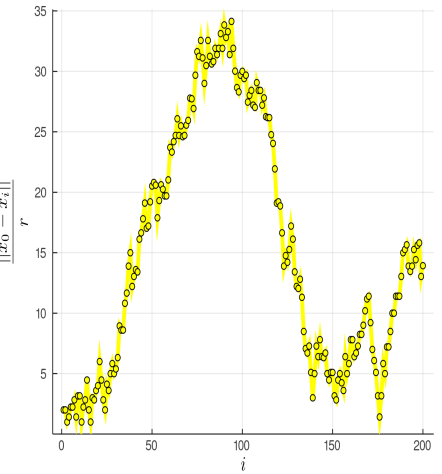

i) Sequence of distances for guigna 9

Figure 7: Graphs of some sequences of distances $\left\{\frac{\left\|x_{0}-x_{i}\right\|}{r}\right\}_{i=1}^{M}$ for different individuals of L. guigna.

The objective of this simulation is to generate a distribution of points in a given territory, in order to emulate radiotracking data in a way analogous to that shown in reference [7]. So, considering an iteration function of a random walker $\Phi_{r}$ that fulfills the following condition

$$
\Phi_{r} \in \operatorname{Gen}_{m}\left(x_{0}\right)
$$

it is possible to generate multiple finite sequences of random variables $\left\{x_{i}\right\}_{i=1}^{m}$ from the same initial condition $x_{0}$, that is,

$$
\left\{x_{i_{1}}\right\}_{i_{1}=1}^{m},\left\{x_{i_{2}}\right\}_{i_{2}=1}^{m}, \cdots,\left\{x_{i_{N}}\right\}_{i_{N}=1}^{m},
$$

which subsequently may be concatenated to generate one sequence of random variables as follows 
Applied Mathematics and Sciences: An International Journal (MathSJ) Vol.8, No.2/3/4, December 2021

$$
\left\{\left\{x_{i_{1}}\right\}_{i_{1}=1}^{m},\left\{x_{i_{2}}\right\}_{i_{2}=1}^{m}, \cdots,\left\{x_{i_{N}}\right\}_{i_{N}=1}^{m}\right\}:=\left\{x_{i}\right\}_{i=1}^{M} \text {. }
$$

So, through 50 sequences of random variables and using the previous process, the distributions of points shown in Figure 8 were generated. They are analogous to distributions as could be obtained by radiotracking field studies as well as the positions of domestic cats as shown in reference [7].

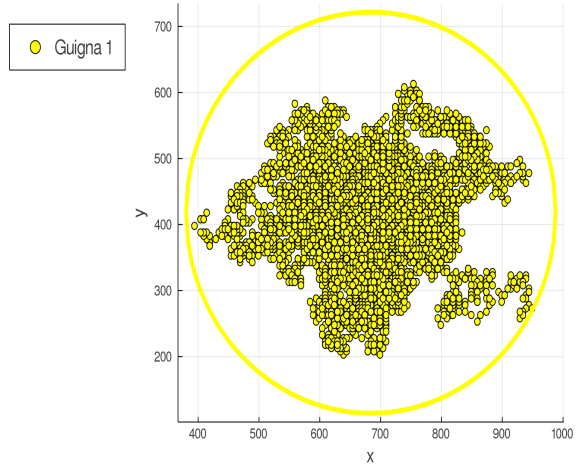

a) Positions of guigna 1

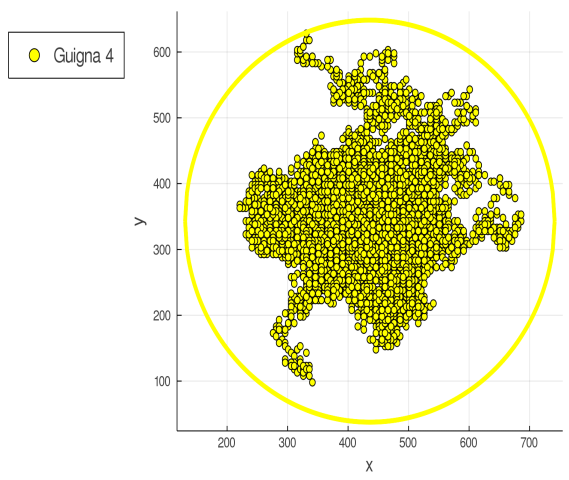

d) Positions of guigna 4

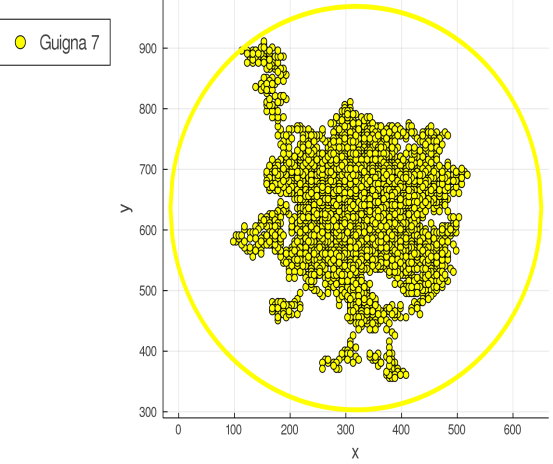

g) Positions of guigna 7

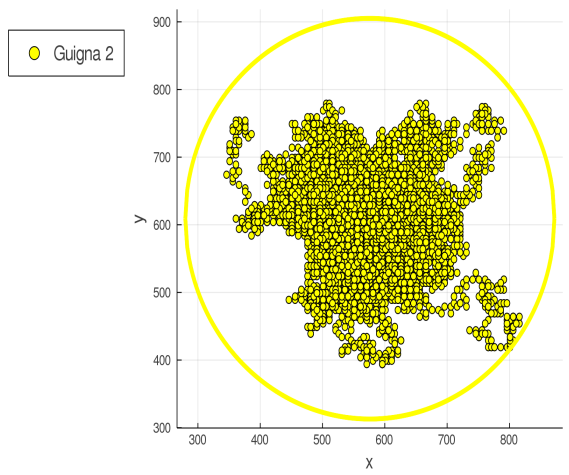

b) Positions of guigna 2
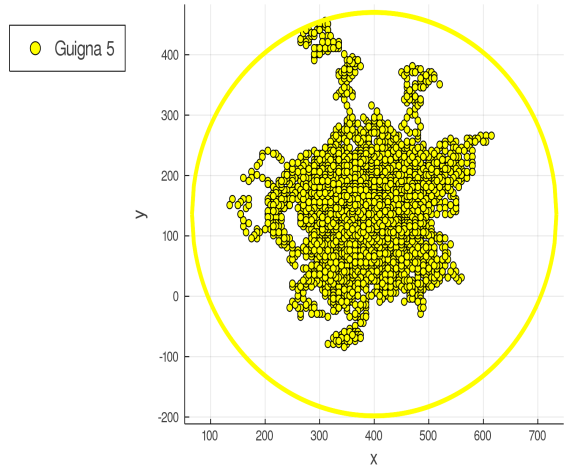

e) Positions of guigna 5

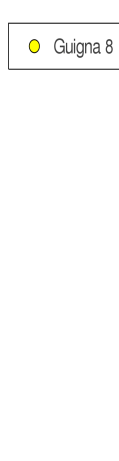

h) Positions of guigna 8

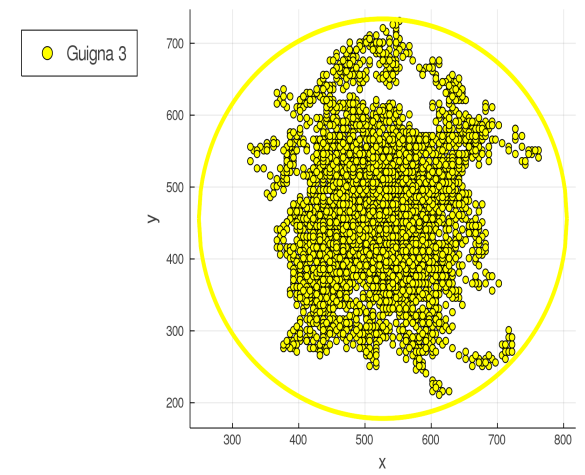

c) Positions of guigna 3

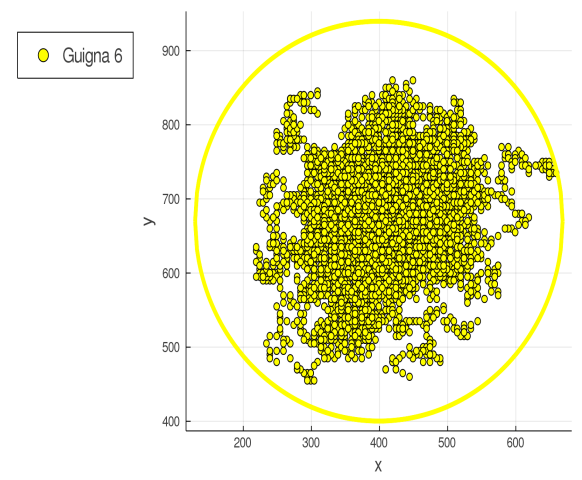

f) Positions of guigna 6
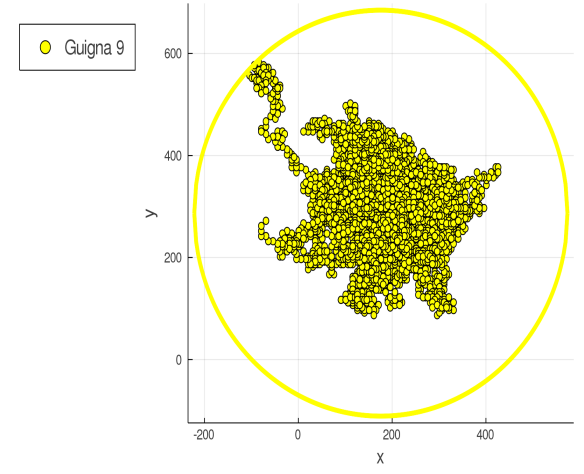

i) Positions of guigna 9

Figure 8: Distributions of positions of different individuals of L. guigna.

Considering that in the simulation each individual of L. guigna generates a distribution of positions given by the following succession of random variables

$$
\left\{x_{i}\right\}_{i=1}^{M} \quad \text { with } \quad M=10^{4},
$$

it is possible to characterize the distances that each guigna moves away from its initial position $x_{0}$ by the following succession of distances in terms of the step size $r$ 


$$
\left\{\frac{\left\|x_{0}-x_{i}\right\|}{r}\right\}_{i=1}^{M} .
$$

So, The distance covered by each guigna with respect to the associated initial position $x_{0}$ may be analyzed using histograms and box plots as shown in Figure 9 and Figure 10, respectively.

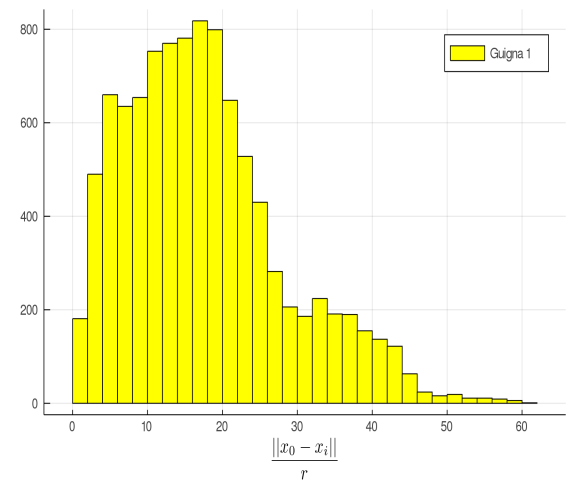

a) Histogram of distances for guigna 1

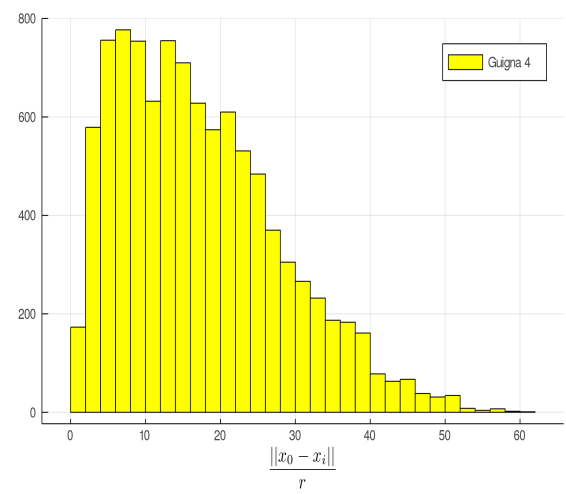

d) Histogram of distances for guigna 4

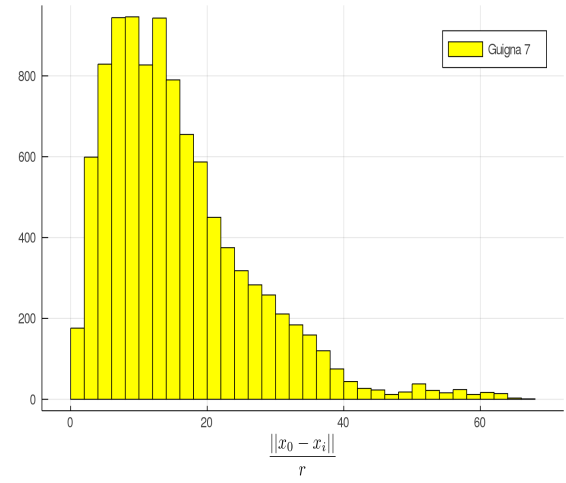

g) Histogram of distances for guigna 7

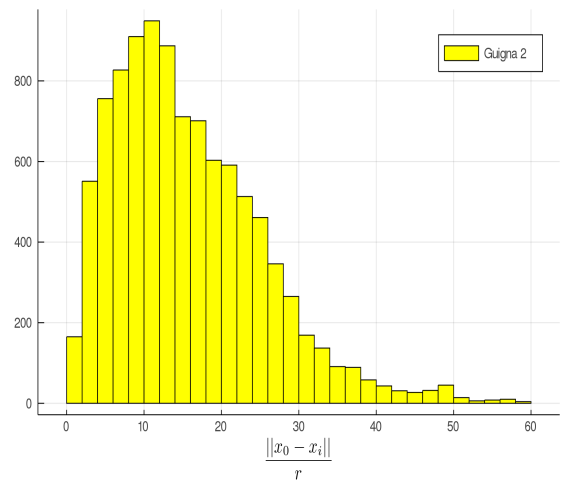

b) Histogram of distances for guigna 2

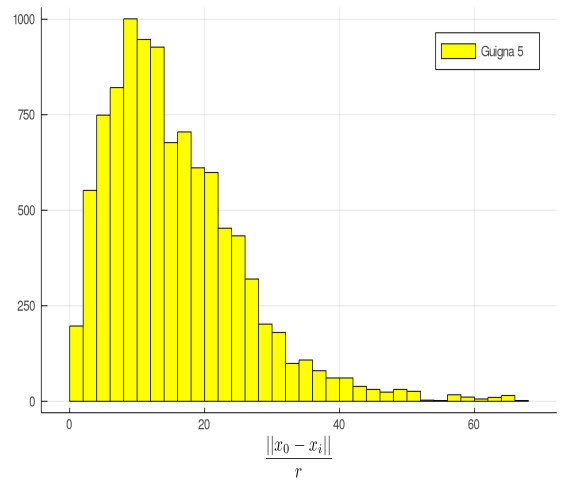

e) Histogram of distances for guigna 5

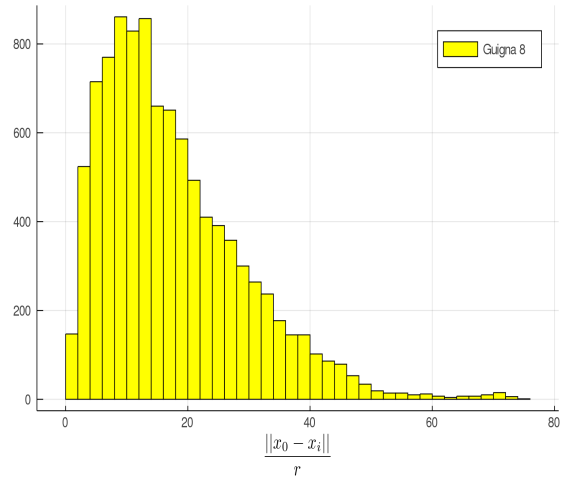

h) Histogram of distances for guigna 8

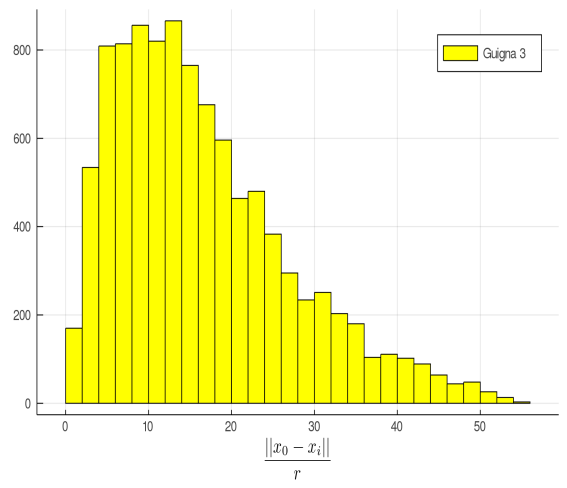

c) Histogram of distances for guigna 3

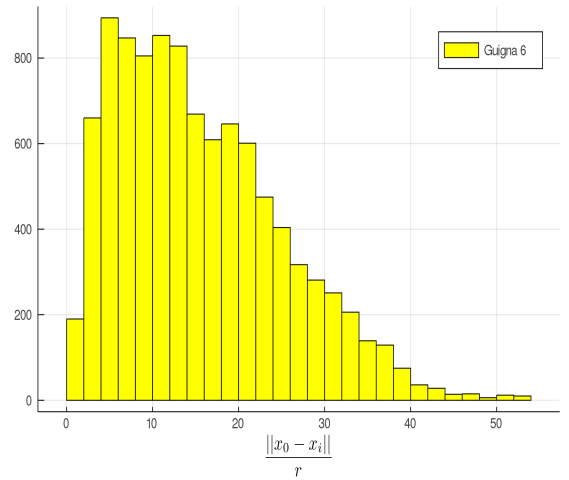

f) Histogram of distances for guigna 6

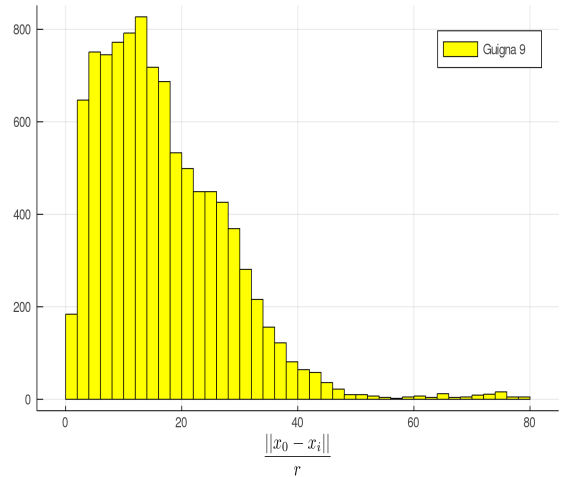

i) Histogram of distances for guigna 9

Figure 9: Histograms of sequences of distances $\left\{\frac{\left\|x_{0}-x_{i}\right\|}{r}\right\}_{i=1}^{M}$ for different individuals of L. guigna . 


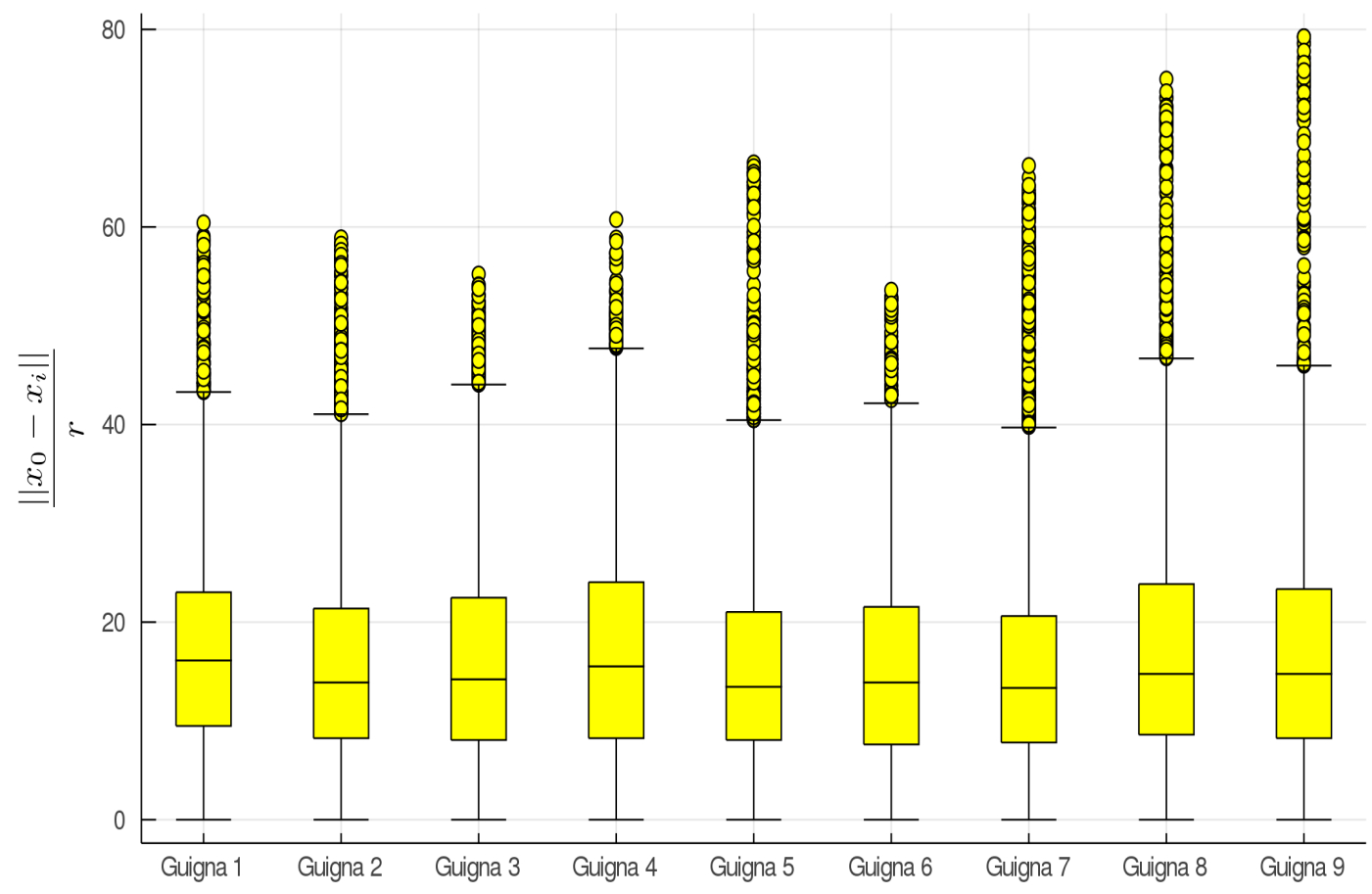

Figure 10: Box plots of sequences of distances $\left\{\frac{\left\|x_{0}-x_{i}\right\|}{r}\right\}_{i=1}^{M}$ of different individuals of L. guigna.

It is necessary to mention that from Figure 10 it may be concluded that the values of the distributions of the distances of the guignas in the simulation have a high level of dispersion, so that the mean distances of the distributions would not be representative measures of the data, since these mean values tend to be affected in the presence of anomalous data caused by dispersion. On the other hand, the medians of the distributions are not considerably affected by the dispersion, so in the presence of data with a high level of dispersion it could be considered as an acceptable measure to characterize the behavior of the data. It is important to mention the above, because the behavior of the distances that could be obtained from tracking different guignas in their territory should not necessarily be uniform, since irregularities may occur due to interactions with external agents or irregularities of the terrain, so work with data with a considerable degree of dispersion is something that falls under the criteria of the simulation, and as a consequence, it may be considered that the most representative measures of the distributions of the data of the guignas generated in the simulation are those shown in the following table:

\begin{tabular}{|c|c|c|c|}
\hline Guignas & $\max _{i \geq 1}\left\{\frac{\left\|x_{0}-x_{i}\right\|}{r}\right.$ & $\operatorname{mean}_{i \geq 1}\left\{\frac{\left\|x_{0}-x_{i}\right\|}{r}\right.$ & $\underset{i \geq 1}{\operatorname{median}}\left\{\frac{\left\|x_{0}-x_{i}\right\|}{r}\right\}$ \\
\hline Guigna 1 & 60.42 & 17.47 & 16.12 \\
\hline Guigna 2 & 58.90 & 15.57 & 13.89 \\
\hline Guigna 3 & 55.23 & 16.41 & 14.21 \\
\hline Guigna 4 & 60.75 & 17.23 & 15.52 \\
\hline Guigna 5 & 66.48 & 15.52 & 13.45 \\
\hline Guigna 6 & 53.60 & 15.42 & 13.89 \\
\hline Guigna 7 & 66.22 & 15.58 & 13.34 \\
\hline Guigna 8 & 74.97 & 17.38 & 14.76 \\
\hline Guigna 9 & 79.25 & 16.71 & 14.76 \\
\hline
\end{tabular}

Table 3: Most representative distances of the sequences of distances $\left\{\frac{\left\|x_{0}-x_{i}\right\|}{r}\right\}_{i=1}^{M}$ of different individuals of L. guigna.

From the previous table it is possible to define the following values for each distribution of positions of the guignas

$$
R_{M}:=\max _{i \geq 1}\left\{\frac{\left\|x_{0}-x_{i}\right\|}{r}\right\}
$$


Applied Mathematics and Sciences: An International Journal (MathSJ) Vol.8, No.2/3/4, December 2021

$$
r_{m}:=\min \left\{\operatorname{mean}_{i \geq 1}\left\{\frac{\left\|x_{0}-x_{i}\right\|}{r}\right\}, \underset{i \geq 1}{\operatorname{median}}\left\{\frac{\left\|x_{0}-x_{i}\right\|}{r}\right\}\right\}
$$

with which it is possible to measure the interactions they have with predators through the following result:

$$
\text { If } \frac{\left\|x_{0}-x_{p}\right\|}{r} \leq R_{M} \text { and }\left\{x_{i}\right\}_{i=1}^{M} \cap B\left(x_{p} ; \frac{r_{m}}{2}\right) \neq \emptyset \Rightarrow \exists\left\{x_{i_{p}}\right\}_{i_{p}=1}^{m} \in B\left(x_{p} ; \frac{r_{m}}{2}\right) \text {, }
$$

where $x_{p}$ denotes the position of some predator, and the values $x_{i_{p}}$ represent positions of the guignas that could be considered dangerously close to some predator. On the other hand, through the values $x_{i_{p}}$ it is possible to characterize the number of interactions that each guigna has with some predators as shown in the following table:

\begin{tabular}{l|ccccccccc}
\hline & Guigna 1 & Guigna 2 & Guigna 3 & Guigna 4 & Guigna 5 & Guigna 6 & Guigna 7 & Guigna 8 & Guigna 9 \\
\hline Predator 1 & 8 & 0 & 0 & 0 & 0 & 0 & 0 & 0 & 0 \\
Predator 2 & 40 & 0 & 0 & 0 & 0 & 0 & 0 & 0 & 0 \\
Predator 3 & 97 & 0 & 72 & 16 & 18 & 0 & 0 & 0 & 0 \\
Predator 4 & 0 & 0 & 0 & 0 & 13 & 0 & 0 & 0 & 0 \\
Predator 5 & 0 & 0 & 14 & 302 & 165 & 0 & 0 & 0 & 0 \\
Predator 6 & 0 & 75 & 0 & 0 & 0 & 464 & 104 & 0 & 0 \\
Predator 7 & 0 & 240 & 70 & 0 & 0 & 1232 & 190 & 53 & 0 \\
Predator 8 & 0 & 39 & 72 & 43 & 0 & 411 & 274 & 272 & 0 \\
Predator 9 & 0 & 0 & 37 & 32 & 0 & 47 & 54 & 595 & 0 \\
Predator 10 & 0 & 0 & 0 & 321 & 36 & 0 & 20 & 3 & 27 \\
Predator 11 & 0 & 0 & 0 & 333 & 0 & 0 & 0 & 0 & 653 \\
Predator 12 & 0 & 0 & 0 & 95 & 161 & 0 & 0 & 0 & 209 \\
Predator 13 & 0 & 0 & 0 & 22 & 201 & 0 & 0 & 0 & 141 \\
Predator 14 & 0 & 0 & 0 & 0 & 61 & 0 & 0 & 0 & 17 \\
\hline
\end{tabular}

Table 4: Number of interactions of different individuals of L. guigna with different predators.

So, considering the following result:

$$
\text { If }\left\{x_{i}\right\}_{i=1}^{m} \in B\left(x_{p} ; \frac{r_{m}}{2}\right) \Rightarrow \forall x_{j} \in\left\{x_{i}\right\}_{i=1}^{m} \exists k_{j}=\frac{\left\|x_{p}-x_{j}\right\|}{r},
$$

\begin{tabular}{|c|c|c|}
\hline Guiga 1 & & \\
\hline Predators & $\operatorname{mean}_{i \geq 1}\left\{\frac{\left\|x_{p}-x_{i}\right\|}{r}\right.$ & $\underset{i \geq 1}{\operatorname{median}}\left\{\frac{\left\|x_{p}-x_{i}\right\|}{r}\right.$ \\
\hline Predator 1 & 7.28 & 7.32 \\
\hline Predator 2 & 6.86 & 6.84 \\
\hline Predator 3 & 5.06 & 5.40 \\
\hline
\end{tabular}

it is possible to analyze the distribution of distances of each guigna with each of the predators with which it interacted as shown below:

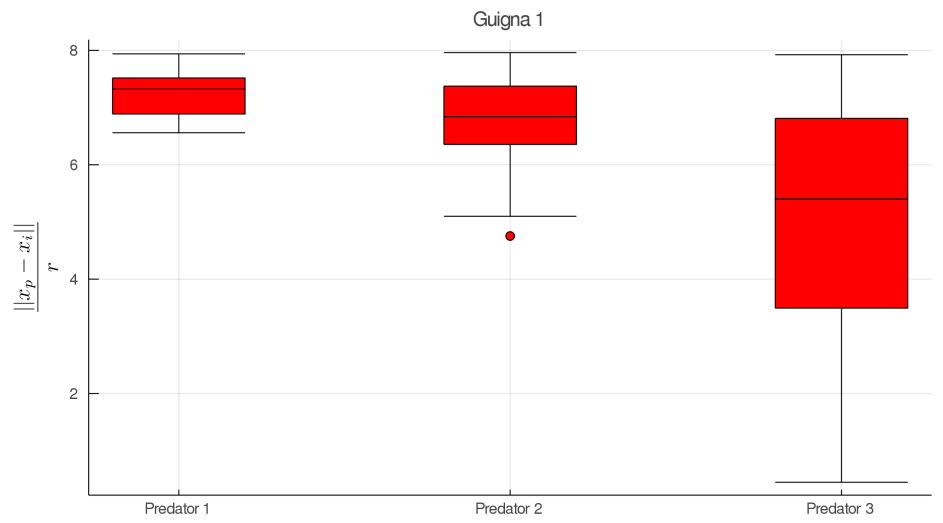

Figure 11: Most representative distances of the interactions of guigna 1 with some predators and box plot of the distances $\left\{\frac{\left\|x_{p}-x_{i}\right\|}{r}\right\}_{i=1}^{m}$ of the interactions of guigna 1 with some predators. 


\begin{tabular}{|c|c|c|}
\hline Guiga 2 & & \\
\hline Predators & $\operatorname{mean}_{i \geq 1}\left\{\frac{\left\|x_{p}-x_{i}\right\|}{r}\right.$ & $\underset{i \geq 1}{\operatorname{median}}\left\{\frac{\left\|x_{p}-x_{i}\right\|}{r}\right.$ \\
\hline Predator 6 & 5.12 & 5.80 \\
\hline Predator 7 & 4.86 & 5.20 \\
\hline Predator 8 & 5.64 & 5.60 \\
\hline
\end{tabular}

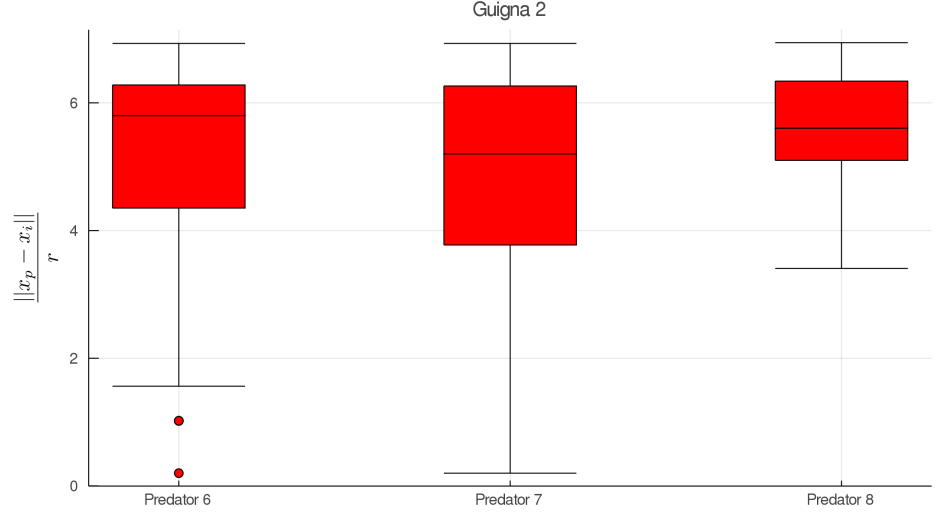

Figure 12: Most representative distances of the interactions of guigna 2 with some predators and box plot of the distances $\left\{\frac{\left\|x_{p}-x_{i}\right\|}{r}\right\}_{i=1}^{m}$ of the interactions of guigna 2 with some predators.

\begin{tabular}{|c|c|c|}
\hline Guiga 3 & & \\
\hline Predators & $\operatorname{mean}_{i \geq 1}\left\{\frac{\left\|x_{p}-x_{i}\right\|}{r}\right.$ & $\underset{i \geq 1}{\operatorname{median}}\left\{\frac{\left\|x_{p}-x_{i}\right\|}{r}\right.$ \\
\hline Predator 3 & 4.32 & 4.29 \\
\hline Predator 5 & 5.88 & 5.92 \\
\hline Predator 7 & 4.59 & 4.71 \\
\hline Predator 8 & 5.42 & 5.75 \\
\hline Predator 9 & 5.45 & 5.73 \\
\hline
\end{tabular}

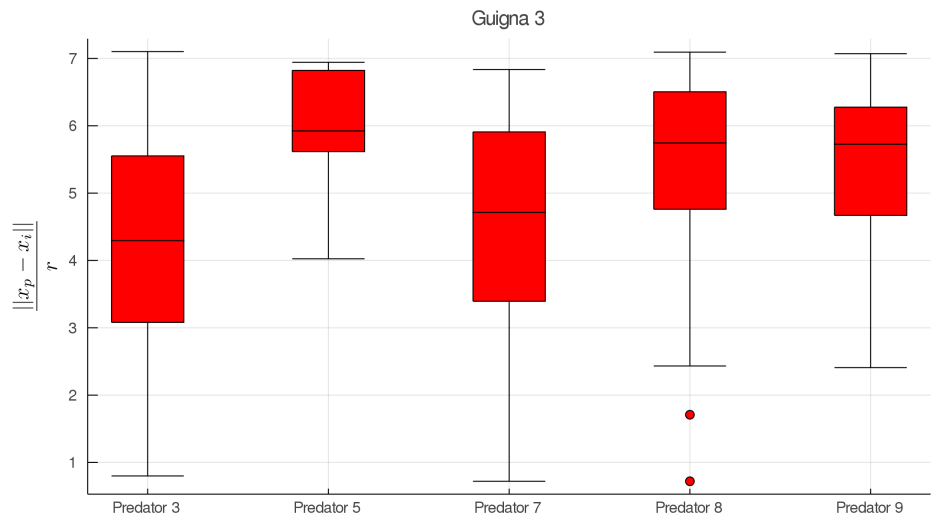

Figure 13: Most representative distances of the interactions of guigna 3 with some predators and box plot of the distances $\left\{\frac{\left\|x_{p}-x_{i}\right\|}{r}\right\}_{i=1}^{m}$ of the interactions of guigna 3 with some predators.

\begin{tabular}{c|cc}
\hline Guiga 4 & \multicolumn{3}{|c}{} \\
\hline Predators & $\operatorname{mean}_{i \geq 1}\left\{\frac{\left\|x_{p}-x_{i}\right\|}{r}\right\}$ & $\begin{array}{c}\text { median } \\
i \geq 1\end{array}\left\{\frac{\left\|x_{p}-x_{i}\right\|}{r}\right\}$ \\
\hline Predator 3 & 6.12 & 6.15 \\
Predator 5 & 5.16 & 5.35 \\
Predator 8 & 5.71 & 6.05 \\
Predator 9 & 6.80 & 7.21 \\
Predator 10 & 5.01 & 5.56 \\
Predator 11 & 4.81 & 5.00 \\
Predator 12 & 5.28 & 5.40 \\
Predator 13 & 4.71 & 4.67 \\
\hline
\end{tabular}

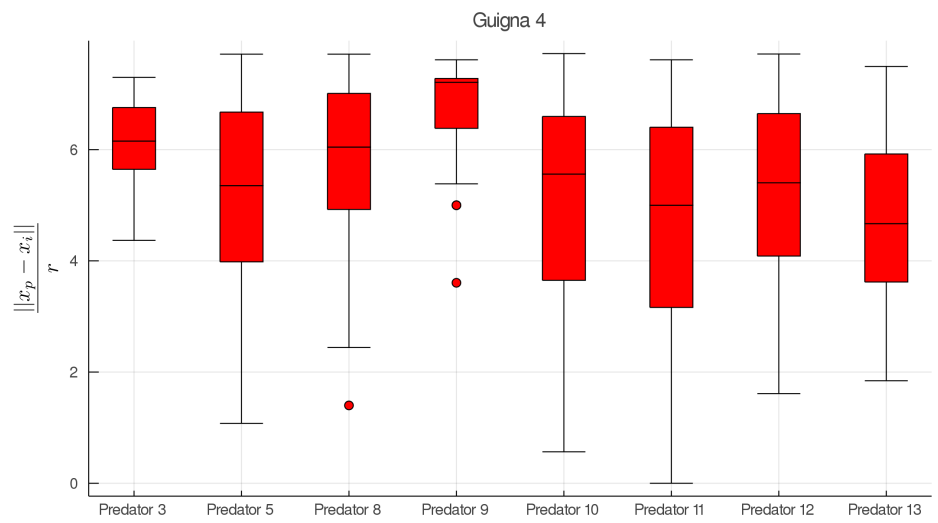

Figure 14: Most representative distances of the interactions of guigna 4 with some predators and box plot of the distances $\left\{\frac{\left\|x_{p}-x_{i}\right\|}{r}\right\}_{i=1}^{m}$ of the interactions of guigna 4 with some predators. 


\begin{tabular}{|c|c|c|}
\hline Guiga 5 & & \\
\hline Predators & $\operatorname{mean}_{i \geq 1}\left\{\frac{\left\|x_{p}-x_{i}\right\|}{r}\right.$ & $\underset{i \geq 1}{\operatorname{median}}\left\{\frac{\left\|x_{p}-x_{i}\right\|}{r}\right.$ \\
\hline Predator 3 & 3.67 & 3.51 \\
\hline Predator 4 & 4.84 & 5.20 \\
\hline Predator 5 & 4.43 & 4.62 \\
\hline Predator 10 & 5.36 & 5.30 \\
\hline Predator 12 & 4.19 & 4.40 \\
\hline Predator 13 & 4.32 & 4.53 \\
\hline Predator 14 & 5.13 & 5.30 \\
\hline
\end{tabular}

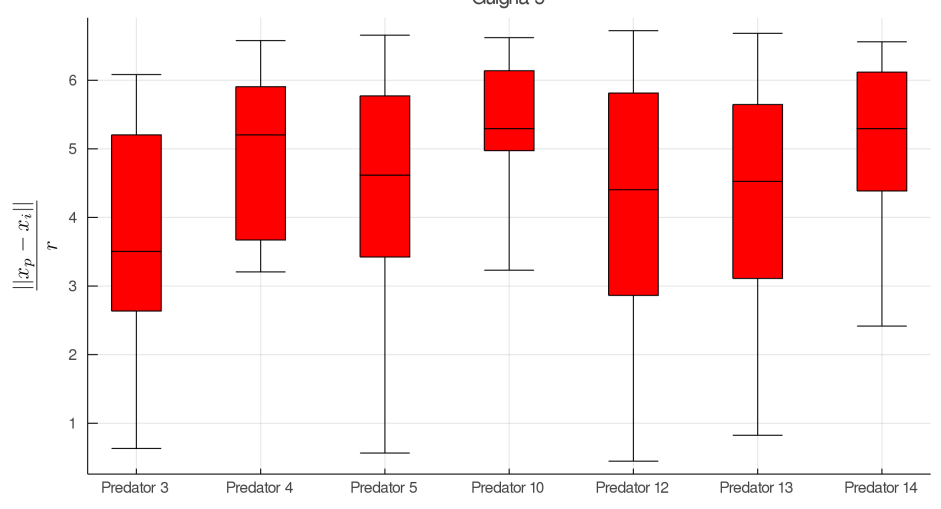

Figure 15: Most representative distances of the interactions of guigna 5 with some predators and box plot of the distances $\left\{\frac{\left\|x_{p}-x_{i}\right\|}{r}\right\}_{i=1}^{m}$ of the interactions of guigna 5 with some predators.

\begin{tabular}{c|cc}
\hline Guiga 6 & & \\
\hline Predators & $\operatorname{mean}_{i \geq 1}\left\{\frac{\left\|x_{p}-x_{i}\right\|}{r}\right\}$ & $\underset{\substack{\operatorname{median} \\
i \geq 1}}{ }\left\{\frac{\left\|x_{p}-x_{i}\right\|}{r}\right\}$ \\
\hline Predator 6 & 4.85 & 5.19 \\
Predator 7 & 4.59 & 4.87 \\
Predator 8 & 4.79 & 5.25 \\
Predator 9 & 5.00 & 5.43 \\
\hline
\end{tabular}

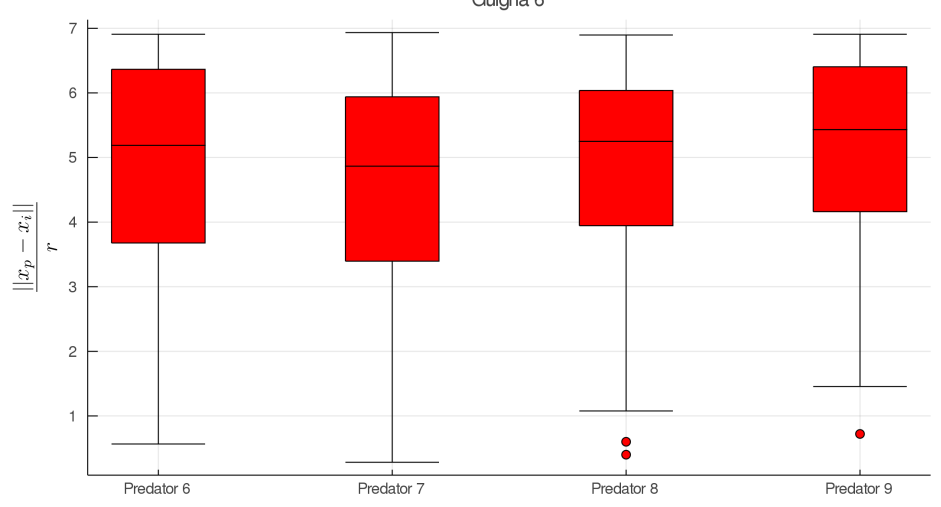

Figure 16: Most representative distances of the interactions of guigna 6 with some predators and box plot of the distances $\left\{\frac{\left\|x_{p}-x_{i}\right\|}{r}\right\}_{i=1}^{m}$ of the interactions of guigna 6 with some predators.

\begin{tabular}{c|cc}
\hline Guiga 7 & & \\
\hline Predators & $\operatorname{mean}_{i \geq 1}\left\{\frac{\left\|x_{p}-x_{i}\right\|}{r}\right\}$ & $\underset{\substack{\text { median } \\
i \geq 1}}{ }\left\{\frac{\left\|x_{p}-x_{i}\right\|}{r}\right\}$ \\
\hline Predator 6 & 4.42 & 4.62 \\
Predator 7 & 4.53 & 4.68 \\
Predator 8 & 4.57 & 4.92 \\
Predator 9 & 5.13 & 5.60 \\
Predator 10 & 4.57 & 4.80 \\
\hline
\end{tabular}

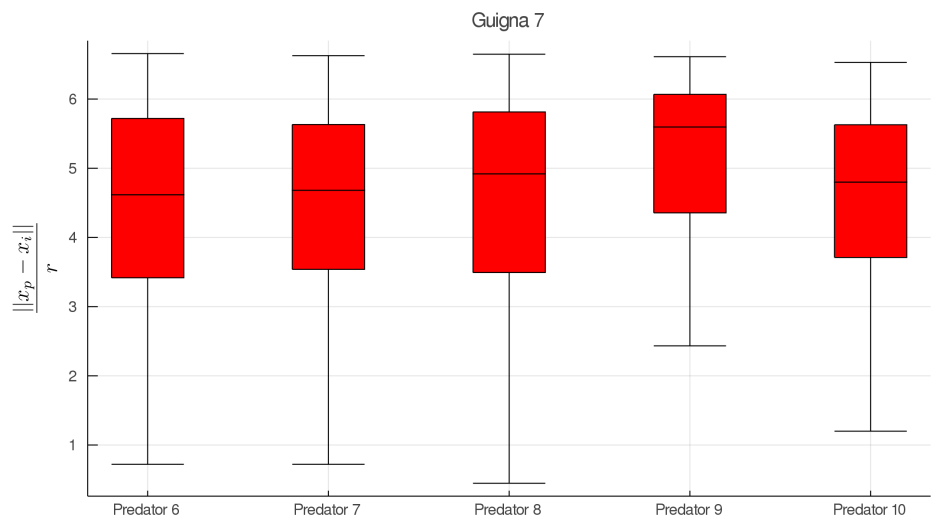

Figure 17: Most representative distances of the interactions of guigna 7 with some predators and box plot of the distances $\left\{\frac{\left\|x_{p}-x_{i}\right\|}{r}\right\}_{i=1}^{m}$ of the interactions of guigna 7 with some predators. 


\begin{tabular}{c|cc}
\hline Guiga 8 & \multicolumn{3}{c}{} \\
\hline Predators & $\operatorname{mean}_{i \geq 1}\left\{\frac{\left\|x_{p}-x_{i}\right\|}{r}\right\}$ & $\underset{\substack{\text { median } \\
i \geq 1}}{ }\left\{\frac{\left\|x_{p}-x_{i}\right\|}{r}\right\}$ \\
\hline Predator 7 & 5.10 & 5.20 \\
Predator 8 & 4.91 & 5.20 \\
Predator 9 & 5.12 & 5.31 \\
Predator 10 & 6.62 & 6.51 \\
\hline
\end{tabular}

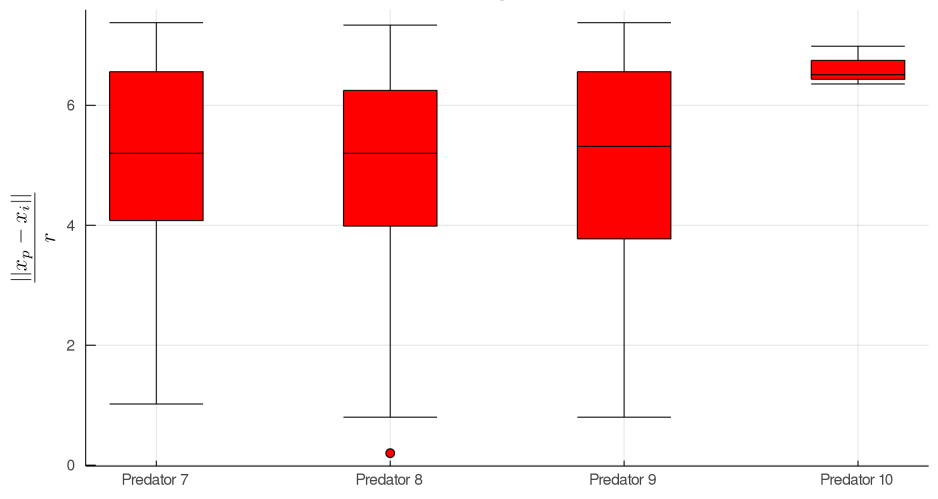

Figure 18: Most representative distances of the interactions of guigna 8 with some predators and box plot of the distances $\left\{\frac{\left\|x_{p}-x_{i}\right\|}{r}\right\}_{i=1}^{m}$ of the interactions of guigna 8 with some predators.

\begin{tabular}{c|cc}
\hline Guiga 9 & & \\
\hline Predators & $\operatorname{mean}_{i \geq 1}\left\{\frac{\left\|x_{p}-x_{i}\right\|}{r}\right\}$ & $\begin{array}{c}\text { median } \\
i \geq 1\end{array}$ \\
\hline Predator 10 & 5.65 & 6.00 \\
Predator 11 & 5.01 & 5.30 \\
Predator 12 & 4.81 & 5.23 \\
Predator 13 & 4.64 & 4.83 \\
Predator 14 & 5.87 & 6.05 \\
\hline
\end{tabular}

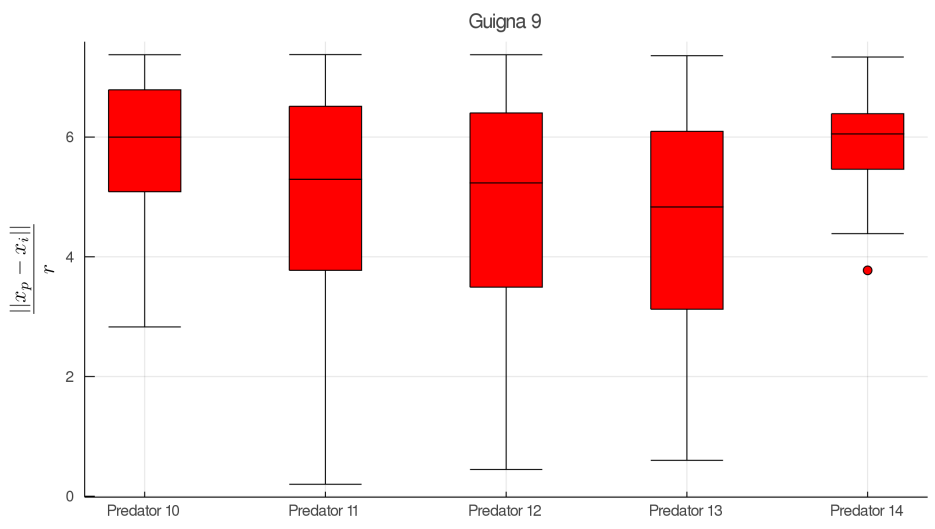

Figure 19: Most representative distances of the interactions of guigna 9 with some predators and box plot of the distances $\left\{\frac{\left\|x_{p}-x_{i}\right\|}{r}\right\}_{i=1}^{m}$ of the interactions of guigna 9 with some predators.

Finally, taking into account the possibility that the data have a high level of dispersion, the following value may be defined

$$
r_{l}:=\min \left\{\operatorname{mean}_{i \geq 1}\left\{\frac{\left\|x_{p}-x_{i}\right\|}{r}\right\}, \underset{i \geq 1}{\operatorname{median}}\left\{\frac{\left\|x_{p}-x_{i}\right\|}{r}\right\}\right\},
$$

with which it is possible to obtain the following result:

$$
\text { If }\left\{x_{i}\right\}_{i=1}^{M} \cap B\left(x_{p} ; r_{l}\right) \neq \emptyset \Rightarrow \text { there exists at least one value } x_{j} \in\left\{x_{i}\right\}_{i=1}^{M} \text {, }
$$

where $x_{j}$ represents a position in which some guigna is at a distance potentially lethal from the predator $x_{p}$.

\section{Conclusions}

After reviewing the available literature, we assumed the hypothesis that L. guigna maintains a sedentary behavior in a specific area of a given territory. Furthermore, we propose a mathematical model which is based on that hypothesis and which was designed to emulate movement data as could be obtained by radiotracking field studies. Therefor, we simulated a distribution of points in a given territory using random walkers. Our approach allows for the estimation of the mean distances covered by L. guigna with respect to certain fixed starting positions, as 
Applied Mathematics and Sciences: An International Journal (MathSJ) Vol.8, No.2/3/4, December 2021

well as the encounters that may occur between individual guignas and potential predators, including other types of threats, such as motor vehicles on roads and people defending their free-ranging poultry. However, due to the shortage of guigna observations, a combination of test data with real data, obtained by camera traps, was used. While our simulation fulfilled its objective, estimates of L. guigna movements could be much improved by the following measures:

i) Establish a coordinate record of possible guigna territories in a region of interest using camera traps.

ii) Establish a coordinate record of predator territories, as well as human activities that pose a threat to $L$. guigna.

iii) Establish a scale in a specific region of interest with confirmed guigna presence from a satellite image.

iv) Establish a step size $r$ considering the scale of the region of interest given in the previous point and the mean distance that individual guignas cover in their home range in a given interval of time such as one day or one week. If guigna-specific data are not available or insufficient, data from other felids showing similar movement behavior could be used.

In this context, our model could be fed with real data whose acquisition is non-invasive and less expensive than true radiotracking studies. One way to collect such data is through citizen science studies that gather reports on direct observations, but the analysis of trap camera images is also a powerful tool. Depending on the quality of the images, it may even be possible to identify individual animals. The inclusion of such data in our model would increase the accuracy of predictions on guigna movement behavior without the need to manipulate live animals. These predictions could then be used to infer the likelihood of encounters between guignas and their threats, which, in turn, opens up possibilities to define priority areas for the implementation of mitigation actions. An analogous approach could be taken to support conservation efforts aimed at protecting other species that show a similarly sedentary behavior as L. guigna.

Acknowledgments: We would like to thank Francisca Javiera Miranda Orcaistegu, who obtained and provided the photographic records of L. guigna. Trap cameras have been installed in the Chiloé National Park, which is administered by the Chilean National Forest Corporation CONAF. Our thanks go out to every member of CONAF who dedicates their work to the conservation of native species.

\section{REFERENCES}

[1] Constanza Napolitano, Cristian Larraguibel-González, Amancay A Cepeda-Mercado, Pablo Vial, and Jim Sanderson. New records of Leopardus guigna in its northern-most distribution in Chile: implications for conservation. Revista chilena de historia natural, 93(1):1-5, 2020.

[2] Mira M Fleschutz, Nicolás Gálvez, Guy Pe’er, Zoe G Davies, Klaus Henle, and Elke Schüttler. Response of a small felid of conservation concern to habitat fragmentation. Biodiversity and Conservation, 25(8):1447-1463, 2016.

[3] C Napolitano, N Gálvez, M Bennett, G Acosta-Jamett, and J Sanderson. Leopardus guigna. The IUCN Red List of Threatened Species 2015. e. T15311A50657245, 2015.

[4] Elke Schüttler, Reinhard Klenke, Stephania Galuppo, Rodrigo A Castro, Cristián Bonacic, Jerry Laker, and Klaus Henle. Habitat use and sensitivity to fragmentation in America's smallest wildcat. Mammalian Biology, 86:1-8, 2017.

[5] Nigel Dunstone, Rachel Freer, Gerardo Acosta-Jamett, Leon Durbin, I Wyllie, M Mazzolli, and D Scott. Uso del hábitat, actividad y dieta de la güiña (Oncifelis guigna) en el Parque Nacional Laguna San Rafael, XI Región, Chile. Boletín del Museo Nacional de Historia Natural (Chile), 51:147-158, 2002.

[6] Jim Sanderson, Mel E Sunquist, and Agustin W Iriarte. Natural History and Landscape-Use of Guignas (Oncifelis guigna) on Isla Grande de Chiloé, Chile. Journal of Mammalogy, 83(2):608-613, 2002.

[7] María José López-Jara, Irene Sacristán, Ariel A Farías, Francisca Maron-Perez, Francisca Acuña, Emilio Aguilar, Sebastián García, Patricio Contreras, Eduardo A Silva-Rodríguez, and Constanza Napolitano. Free-roaming domestic cats near conservation areas in Chile: Spatial movements, human care and risks for wildlife. Perspectives in Ecology and Conservation, 2021. 